DOI: https://doi.org/10.14311/TPFM.2022.015

\title{
ESTIMATING RHEOLOGICAL PROPERTIES OF SUSPENSIONS FORMED OF ARBITRARILY-SHAPED PARTICLES VIA CFD-DEM
}

\author{
Martin Kotouč Šourek ${ }^{1}$, Martin Isoz ${ }^{2,3}$ \\ ${ }^{1}$ Department of Chemical Engineering, Faculty of Chemical Engineering, University of Chem- \\ istry and Technology, Technická 5, Prague 166 28, Czech Republic \\ ${ }^{2}$ Department of Mathematics, Faculty of Chemical Engineering, University of Chemistry and \\ Technology, Technická 5, Prague 166 28, Czech Republic \\ 3 Czech Academy of Sciences, Institute of Thermomechanics, Dolejškova 5, Prague 18200 , \\ Czech Republic
}

\begin{abstract}
In recent years, new methods combining computational fluid dynamics (CFD) and discrete element method (DEM) have been intensively studied. Usually, these methods are focused on simulations of spherical particles. Nevertheless, this is inadequate for a simulation of a common suspension, the rheology of which is affected by particle shapes. In this work, we leverage the capabilities of an in-house developed CFD-DEM solver to simulate suspensions formed of arbitrarily-shaped particles. Specifically, we simulate a rheological measurement to estimate the suspension viscosity. The CFD-DEM estimates are in very good agreement with available experimental data and correlations proving the new solver capabilities regarding firstprinciples-based simulations of complex non-Newtonian suspension behaviour. The practical potential of suspension simulation is illustrated in a numerical study of the washcoating process in the preparation of a catalytic filter for automotive exhaust gas after-treatment.
\end{abstract}

Keywords: CFD-DEM, HFDIB, suspension rheology, OpenFOAM.

\section{Introduction}

Particulate suspensions play significant roles in many real-life and industrial processes. As a few examples, consider sediment transport in river beds, blood cells motion in vessels, fluidized beds or coating of a porous substrate by a catalytically active material. Due to the importance of processes involving suspensions, various experimental and numerical efforts were made to understand the behaviour of these systems $[3,6]$.

The credibility of any numerical simulation of a particulate suspension stands on a reliable model for the suspension itself. The simplest commonly used model for CFD simulations of suspensions is based on the so-called Eulerian-Eulerian approach, in which the solid phase is approximated as a fluid with an effective density and viscosity [5]. Because this approach is comparatively computationally cheap, it is a good choice for the first insight into the process, or for large-scale simulations. However, the Eulerian-Eulerian approach cannot capture any local fluid-solid interactions and relies on parameters fitted to experimental data for a specific suspension. An alternative to the Eulerian-Eulerian approach capable of suspension simulation based on first-principles fluidsolid and solid-solid interactions is to couple the computational fluid dynamics (CFD) for the fluid with the discrete element method (DEM) for the solid phase, i.e. a CFD-DEM method [11].

There are two main branches of CFD-DEM methods. First, the unresolved CFD-DEM is based on the volume-averaged Navier-Stokes equations and the used computational mesh is coarser than the size of particles. Therefore, several particles can be present in the same CFD cell and the solidphase distribution is projected onto the CFD mesh by the solid phase volume fraction. Coupling between the phases is provided through an explicit expression for drag and lift forces acting on the solid particles [4]. Consequently, the drag and lift for each particle shape need to be estimated from a correlation and solid-fluid interactions on the particle-scale cannot be directly modelled. To fully resolve the particle-laden flow on all scales, a resolved CFD-DEM is required. In the resolved CFD-DEM, the solid phase is usually projected onto the CFD mesh utilizing a variant of the immersed boundary method and the fluid-solid coupling is ensured by enforcing the standard no-slip boundary condition at the particles surface [2]. Consequently, the resolved CFD-DEM demands mesh to be finer than the smallest particle in the system, which significantly increases the simulation computational cost. 
Despite the computational costs of the resolved CFD-DEM, it is the only method capable of first-principles based simulations of suspensions comprising arbitrarily shaped solid particles, i.e. of estimating the suspension rheology purely from the material data for the fluid and solid phases and from the particles shapes. For example, as the flow around each particle is fully resolved, the resolved CFD-DEM is able to capture the rotational impact of a fluid on a particle when there is a perceptible difference in a velocity field around the particle, which is an effect that plays a key role in the suspension rheological properties [10].

In the present paper, we utilize an in-house developed solver based on the hybrid fictitious domain-immersed boundary (HFDIB) method and a level-set-like DEM to study suspension flows. Specifically, we performed simulations of rheological properties of suspensions which differed in solid phase volume fraction and in the shape of dispersed particles. First, we validated the proposed simulation setup via estimating the viscosity of a suspension made of spherical particles and comparing the estimates against available correlations and experimental data $[1,7,8]$. Afterwards, we investigated suspensions comprising non-spherical particles. Please note, that there is no sufficient correlation for the viscosity of such suspensions and only experimental data by Mueller et al. [8] were available for comparison. Moreover, we used the solver for a simulation of a suspension flow through a porous medium. This serves as an illustration of a possible solver usage for a numerical study of the washcoating process [3].

\section{Computational methodology}

There are two principal questions that have to be answered when a common CFD solver should be extended for a generally moving solid phase as depicted in Fig. 1.

1. How include the solid phase in a computational domain to avoid frequent remeshing?

2. How describe the movement of the solid phase?

In our in-house developed CFD-DEM solver, we have utilized an HFDIB method to resolve the first question $[2,9,11]$. To describe particles motion, we incorporated a variant of DEM that is able to account for arbitrarily-shaped particles [11]. The whole solver is implemented in the framework of open-source software OpenFOAM.

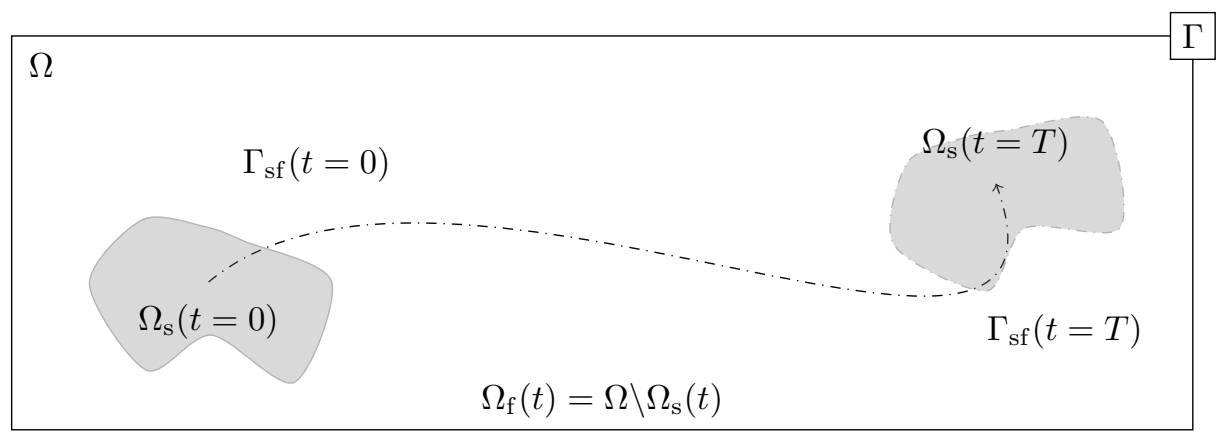

Figure 1: Solution domain $\Omega$ and its division into $\Omega_{\mathrm{s}}, \Omega_{\mathrm{f}}$ and $\Gamma_{\mathrm{sf}}$ at two different times $t=0$ and $t=T$. Movement of $\Omega_{\mathrm{s}}$ in $\Omega$ is illustrated by a dash-dotted line.

\subsection{HFDIB method}

At each time $t$, the distribution of the solid phase is projected into the computational domain via a discrete indicator function $\lambda$ defined as,

$$
\lambda=\left\{\begin{array}{rll}
0 & \text { in } & \Omega_{\mathrm{f}} \\
1 & \text { in } & \Omega_{\mathrm{s}} \\
\tilde{\lambda} \in(0,1) & \text { in } & \Gamma_{\mathrm{sf}}
\end{array},\right.
$$

where $\Omega_{\mathrm{f}}$ represents the part of the computational domain $\Omega$ that is occupied by a fluid, $\Omega_{\mathrm{s}}$ is the part occupied by a solid phase and $\Gamma_{\text {sf }}$ is the fluid-solid interface. Please bear in mind, that 
this projection does not preserve the sharp edges of particles. The considered flow governing equations correspond to the standard variant of laminar Navier-Stokes equations for an incompressible Newtonian fluid with additional forcing term $s$,

$$
\begin{gathered}
\frac{\partial \boldsymbol{u}}{\partial t}+\nabla \cdot(\boldsymbol{u} \otimes \boldsymbol{u})-\nabla \cdot(\nu \nabla \boldsymbol{u})=-\nabla \tilde{p}+\boldsymbol{g}+\boldsymbol{s}, \\
\nabla \cdot \boldsymbol{u}=0
\end{gathered}
$$

where $\boldsymbol{u}$ is the fluid velocity, $\nu$ kinematic viscosity, $\tilde{p}$ kinematic pressure and $\boldsymbol{g}$ the gravitational acceleration. The forcing term $s$ is constructed in a way that it generates a fictitious representation of the solid phase in the computational domain.

$$
s=\left\{\begin{array}{ll}
\frac{\boldsymbol{u}_{\mathrm{IB}}-\boldsymbol{u}^{n}}{\triangle t}+\nabla \cdot\left(\boldsymbol{u}^{n} \otimes \boldsymbol{u}^{n}\right)-\nabla \cdot \mathbf{T}^{n}+\nabla p^{n} & \text { in } \Omega_{\mathrm{s}} \cup \Gamma_{\mathrm{sf}} \\
0 & \text { in } \Omega_{\mathrm{f}}
\end{array},\right.
$$

This approach is known as the hybrid fictitious domain-immersed boundary method and is based on the works Blais et al. [2] and Municchi and Radl [9].

To ensure coupling between phases, the no-slip boundary condition has to be satisfied at the particle surface. This is not straightforward because the surface point $S$ is not always collocated with the mesh point $C$ where the information about velocity is held as depicted in Fig. 2.

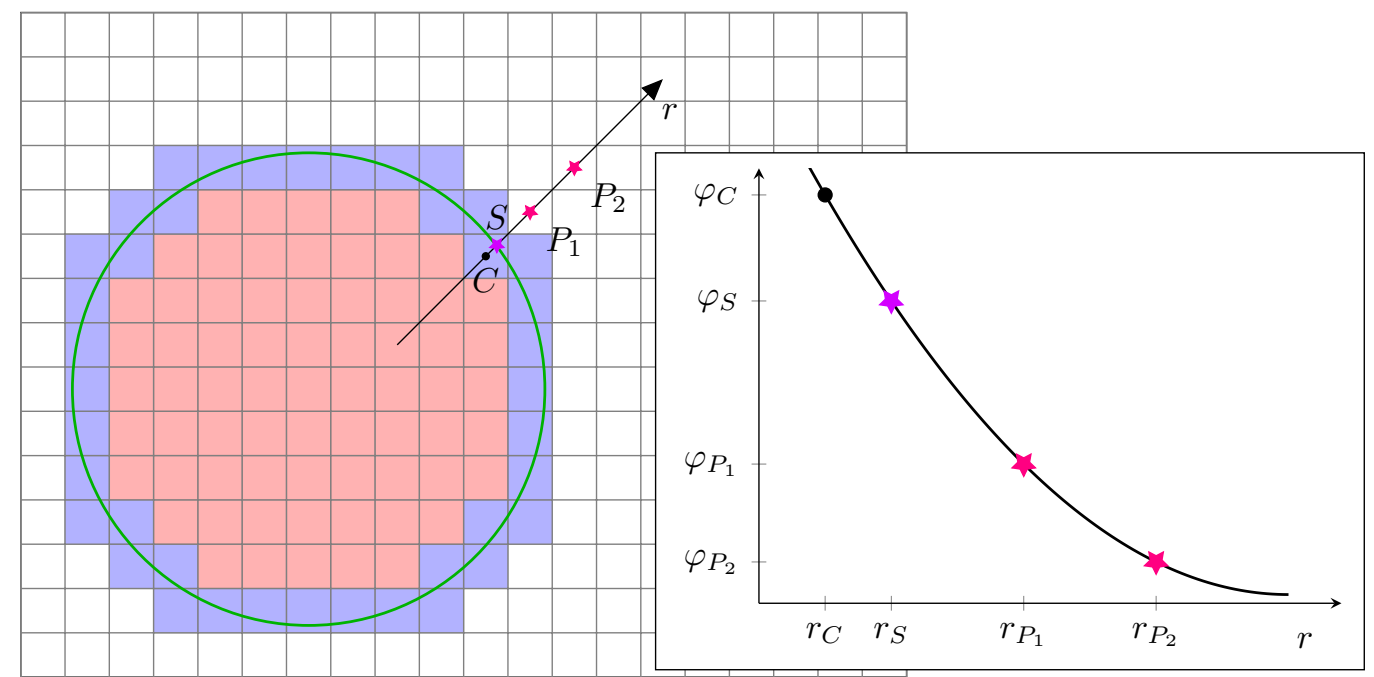

Figure 2: Principle of the reconstruction of an arbitrary intensive tensorial quantity $\varphi$ on the solid-fluid interface. $\Omega_{\mathrm{f}}$ is in white, $\Omega_{\mathrm{s}}$ in red and $\Gamma_{\mathrm{sf}}$ in blue.

To overcome this issue, velocity has to be reconstructed in $\Gamma_{\mathrm{sf}}$. For any given intensive tensorial quantity $\varphi$, we compute the value $\varphi_{C}:=\varphi(C)$ using a second-order polynomial approximation. In particular, a line $r$ orthogonal to $\Gamma_{\text {sf }}$ and passing through the point $S$ is constructed. Next, the values of $\varphi$ at points $\mathrm{P}_{1}$ and $\mathrm{P}_{2}$ lying on $r$ are computed using a suitable interpolation scheme. Finally, $\delta_{1}$ and $\delta_{1}+\delta_{2}$ have to be determined to estimate the value $\varphi_{C}$ from a second-order polynomial as indicated in Fig. 2, i.e.

$$
\begin{gathered}
\varphi_{C} \approx a_{2}\|C-S\|^{2}+b_{2}\|C-S\|+c_{2}, \quad a_{2}:=\frac{\left(\tilde{\varphi}_{P_{2}}-\tilde{\varphi}_{P_{1}}\right) \delta_{1}-\tilde{\varphi}_{P_{1}} \delta_{2}}{\delta_{1} \delta_{2}\left(\delta_{1}+\delta_{2}\right)}, \\
b_{2}:=\frac{\left(\tilde{\varphi}_{P_{1}}-\tilde{\varphi}_{P_{2}}\right) \delta_{1}^{2}+2 \tilde{\varphi}_{P_{1}} \delta_{1} \delta_{2}+\tilde{\varphi}_{P_{1}} \delta_{2}^{2}}{\delta_{1} \delta_{2}\left(\delta_{1}+\delta_{2}\right)}, \quad c_{2}:=\varphi_{S} \\
\tilde{\varphi}_{P_{1}}=\varphi_{P_{1}}-\varphi_{S}, \tilde{\varphi}_{P_{2}}=\varphi_{P_{2}}-\varphi_{S}, \delta_{1}=\left\|S-P_{1}\right\|, \delta_{2}=\left\|P_{1}-P_{2}\right\| .
\end{gathered}
$$

Let us point out that the accuracy of this approximation depends on the correct estimation of point $S$ and normal vector $r$. However, further details on this topic are over the scope of this paper and we refer the reader to our previous work [11]. 


\subsection{DEM method}

DEM is a finite difference numerical method for prediction of the motion of individual and independently moving objects in the Lagrangian framework, i.e. each solid body is treated individually. At any given time $t$, each body is described by its position and angular velocity. Moreover, the movement of the $i$-th body $\left(\mathcal{B}_{i}\right)$ is described by Newton's second law of motion,

$$
m_{i} \frac{\mathrm{d}^{2} \boldsymbol{x}_{i}}{\mathrm{~d} t^{2}}=\sum_{j=1}^{N_{f}} \boldsymbol{f}_{i}^{j}, \quad I_{i} \frac{\mathrm{d} \boldsymbol{\omega}_{i}}{\mathrm{~d} t}=\sum_{j=1}^{N_{f}} \boldsymbol{t}_{i}^{j},
$$

where $m_{i}$ stands for the mass of $\mathcal{B}_{i}$ and $\boldsymbol{x}_{i}(t)$ for its centroid position at time $t$. Further, $\boldsymbol{\omega}_{i}$ is the angular velocity and $I_{i}$ is the matrix of inertial moments. The sums on the right-hand sides of equations (5) represent all the forces $\boldsymbol{f}$ and torques $\boldsymbol{t}$ acting on $\mathcal{B}_{i}$, respectively.

Equations (5) are solved numerically within the finite difference method. During each time step, the translational and angular accelerations are assumed constant $\left(\overline{\boldsymbol{a}}_{i}\right.$ and $\overline{\boldsymbol{\alpha}}_{i}$, respectively) and computed as

$$
\overline{\boldsymbol{a}}_{i}=\frac{1}{m_{i}} \sum_{j=1}^{N_{f}} \boldsymbol{f}_{i}^{j}, \quad \overline{\boldsymbol{\alpha}}_{i}=I_{i}^{-1} \sum_{j=1}^{N_{f}} \boldsymbol{t}_{i}^{j} .
$$

Afterwards, $\overline{\boldsymbol{a}}_{i}$ and $\overline{\boldsymbol{\alpha}}_{i}$ are used to compute incremental changes in the position and rotation of $\mathcal{B}_{i}$.

For further details on DEM implementation, we refer the reader to [11]. However, note that our solver is able to account for arbitrarily-shaped particles and as such, it allows for studies of particle shape impact on the flow. A detailed description of the used DEM extension for arbitrarily-shaped solids is available in [12].

\section{Simulations of suspension rheological properties}

\subsection{Simulation setup}

In this work, we focused on a simulation of a suspension viscosity measurement. The computational domain is designed in order to mimic rheometric equipment. It consists of a three-dimensional box with two parallel solid plates, see Fig. 3. The boundary condition for the moving wall prescribes a velocity of $1 \mathrm{~m} / \mathrm{s}$ along the x-axis. The static wall is not moving and the distance $L$ between plates is $0.1 \mathrm{~mm}$. Furthermore, we defined the zero gradient condition at the geometry outlet and symmetry at the front and back sides of the box.

If there was only fluid in the domain, the simulation setup would correspond to the laminar Couette flow. Thus, we defined a linear velocity profile at the geometry inlet to facilitate the development of the final velocity profile inside the domain. The shear rate between the moving and static walls is evaluated as

$$
\frac{\mathrm{d} \boldsymbol{u}}{\mathrm{d} z}=\frac{\boldsymbol{u}_{\text {Moving wall }}}{L} .
$$

Let us note that no periodic condition for particles is available in the current implementation of our solver. Consequently, when a particle leaves the domain through the outlet, it is not relocated to the corresponding position near the inlet. Instead, particles are randomly added to the zone near the inlet to keep the preset volume fraction of solids. The initial movement of particles is coupled with the fluid velocity field to minimize the domain length required for viscosity estimation. The estimation of the viscosity itself stands on Newton's law of viscosity,

$$
\boldsymbol{\tau}=\nu \frac{\mathrm{d} \boldsymbol{u}}{\mathrm{d} z}
$$

where $\boldsymbol{\tau}$ is shear stress and $\mathrm{d} \boldsymbol{u} / \mathrm{d} y$ is shear rate. The shear stress acting on the moving wall is retrieved from the built-in postprocessing function in OpenFOAM called wallShearStress. However, we retrieve the shear stress only for a part of the moving wall near the outlet to ensure that the correct velocity profile is already reached.

Besides the simulation of a suspension composed of spherical particles, depicted in Fig. 3, we also investigated the impact of the particle shape on the suspension apparent viscosity. Particle shapes were chosen to correspond to the work of Mueller et al. [8] in order to enable comparison 


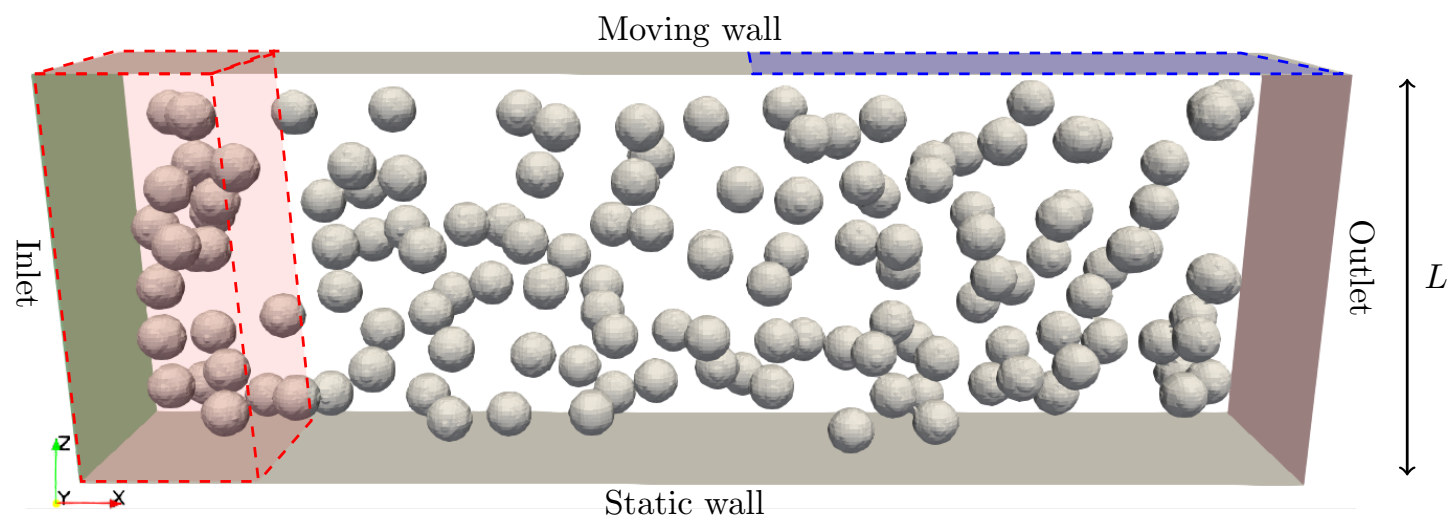

Figure 3: Illustration of the computational domain with a description of important boundaries. The zone for particles addition and the zone for the shear stress measurement are depicted in red and blue, respectively.

between our results and experimental data. Similarly to To describe [8], the particle shape is defined as

$$
r_{\mathrm{p}}=\frac{l_{a}}{l_{b}}
$$

where $l_{a}$ is the particle's axis of rotational symmetry and $l_{b}$ is its maximum diameter perpendicular to that axis. Chosen particles with respective values of $r_{\mathrm{p}}$ are depicted in Fig. 4.

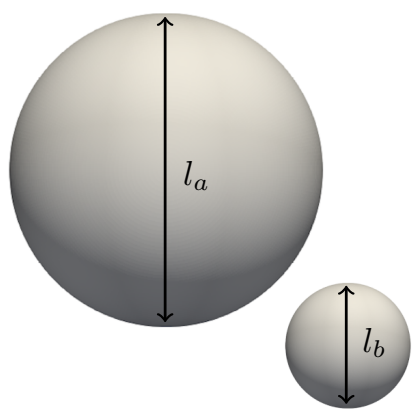

(a) Sphere; $r_{\mathrm{p}}=1$

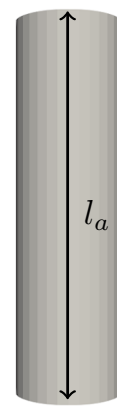

(b) Rod; $r_{\mathrm{p}}=3.5$

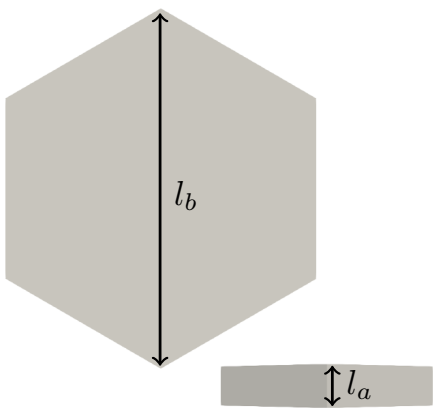

(c) Glitter; $r_{\mathrm{p}}=0.16$

Figure 4: Shapes of suspension particles with depicted characteristic lengths and corresponding values of $r_{\mathrm{p}}$.

\subsection{Results and discussion}

We performed a set of simulations for each particle shape differing in the volume fraction of solids. Suspension made of spherical particles served for validation. Results were compared to available experimental data as well as to commonly used correlations, namely the Batchelor's [1] and Krieger's and Dougherty's correlations [7], respectively:

$$
\nu_{\mathrm{r}}^{\mathrm{B}}=1+2.5 \phi+6.2 \phi^{2}, \quad \nu_{\mathrm{r}}^{\mathrm{KD}}=\left(1-\frac{\phi}{\phi_{m}}\right)^{-2.5 \phi_{m}},
$$

where $\nu_{\mathrm{r}}$ stands for relative viscosity defined as $\nu_{\mathrm{r}}=\frac{\nu}{\nu_{0}}$ and $\nu_{0}$ is the viscosity of pure solvent. Further, $\phi$ represents the volume fraction of solids and $\phi_{m}$ is the maximum packing volume fraction hereafter fixed as $\phi_{m}=0.65$. 

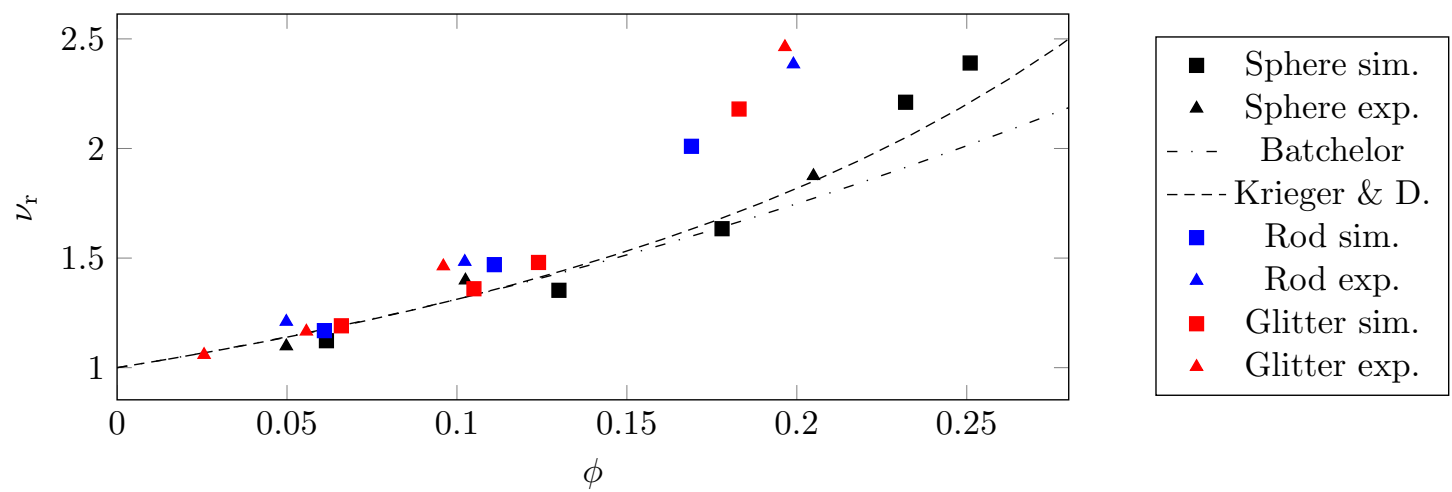

Figure 5: Comparison of estimated and measured suspensions viscosities in dependence on particles volume fraction and shape.

Results computed for the suspensions made of spherical particles are in good agreement with the available data as well as with the commonly used correlations. Moreover, the results for irregularlyshaped particles show similar dependence between relative viscosity and the volume fraction as the experimental data from [8]. The obtained agreement between our method estimates and available data suggests that the method is applicable for numerical investigations of suspensions comprising arbitrarily-shaped particles, which commonly requires an experimental approach.

\section{Numerical study of washcoating process}

To demonstrate the solver capabilities in real-life applications, we performed a numerical study of the washcoating process. The process corresponds to the deposition of the catalytic material inside the porous walls of particulate filters in automotive exhaust gas after-treatment systems. Such a deposition process and its role on the resulting device properties have been recently studied experimentally by Blazek et al. [3].

Here, we simulate numerically the deposition of catalyst in two different sections of a sample investigated by Blazek et al. [3]. The catalytic material is deposited into the filter walls in a form of a water-based slurry. Thus, the washcoating process can be described as a flow of a suspension through a porous structure, i.e. as a process suitable for our CFD-DEM solver. The selected sample sections differed in the morphology of the filter wall. The first section has a large open pore in the middle, whereas the second one is relatively closed. Real samples were scanned by an X-ray microtomography and transformed into a computational domain afterwards, using an OpenFOAM built-in tool called snappyHexMesh. The computational domain was extended by an open space added on top of the porous structure, see Fig. 6b. Such an extension allowed us to define an addition zone for solids similar to the one shown in Fig. 3. Moreover, it provided a buffer for the development of a physical flow velocity profile at the structure inlet. The flow itself is driven by a prescribed pressure difference between the inlet and the outlet.

\subsection{Results and discussion}

Results depicted in Fig. 7 shows a qualitative comparison of the simulated and experimentally obtained distributions of catalytic particles. In Fig. 7a,d, we give an overall view of resulting distributions for a structure with a large open pore and a closed structure, respectively. The corresponding comparison for a single slice through the structure is given in Fig. 7b,c and Fig. 7e,f, respectively.

Comparison between pictures (b) and (e) in Fig. 7 shows the difference in the final coating distribution caused by the different filter wall morphology. For a section with a large pore, the catalytic material is deposited mainly inside the wall. On the other hand, for the relatively closed section, the coating is concentrated predominantly on the wall. This trend corresponds to experimental observations depicted in Fig. 7c,f. The observed qualitative agreement between the simulation and experiment supports the potential future usage of our solver for washcoating simulations. 
(a)

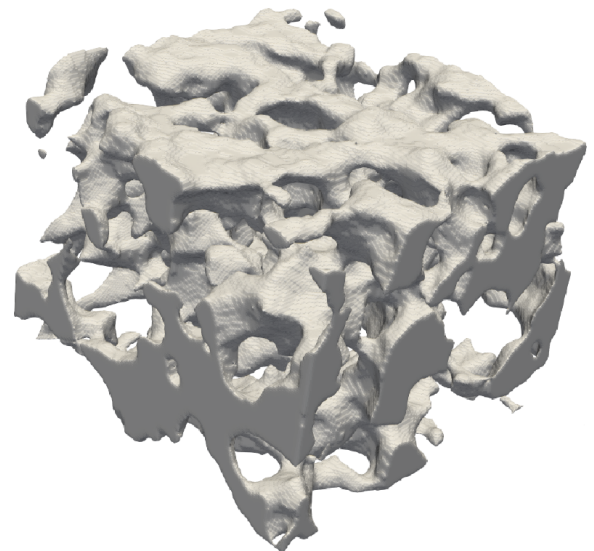

(b)

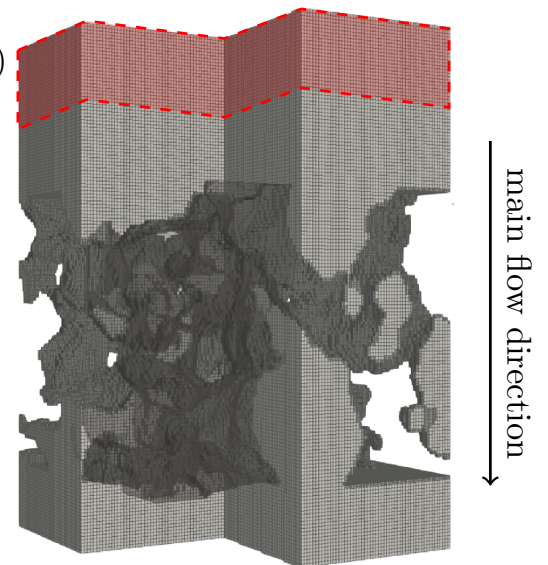

Figure 6: (a) Illustration of a filter wall reconstructed based on XRT data of a real sample. (b) Example of an initial mesh structure and illustration of the zone for solids addition.
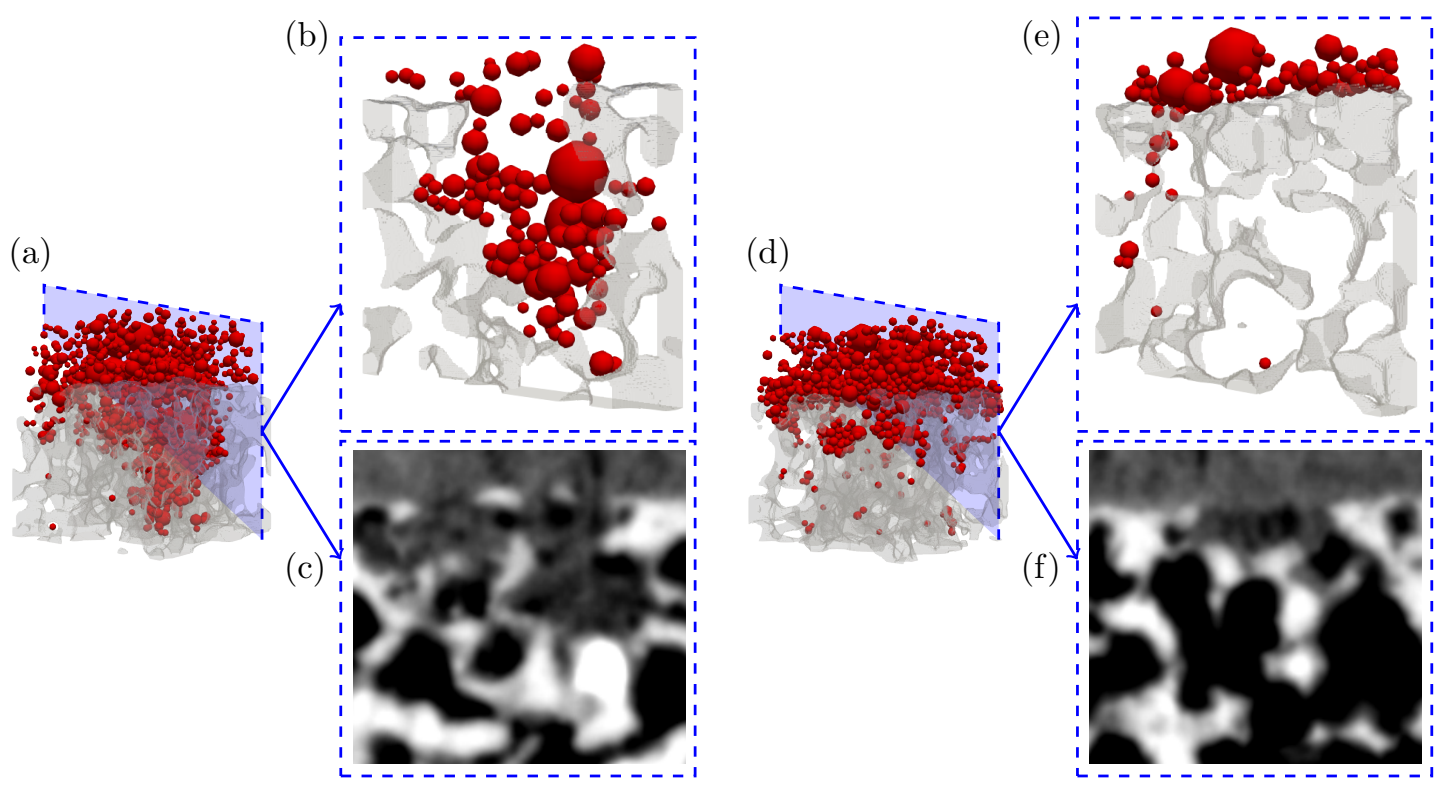

Figure 7: Comparison of the washcoating simulations (a,b,d,e) to the experimental data (c,f) for two different sections of the filter wall: a section with a large pore on $(\mathrm{a}, \mathrm{b}, \mathrm{c})$ and a closed structure $(\mathrm{d}, \mathrm{e}, \mathrm{f})$. Color code for the numerical results: grey $=$ wall, red $=$ catalyst. Color code for the experimental data: white $=$ wall, black $=$ void space, grey $=$ catalyst .

\section{Conclusion}

Fully coupled particle-laden flows are still an interesting topic from the point of view of both experimental and numerical studies. Although measurements of rheological properties of suspensions are great examples of such particle-laden flows, numerical studies are predominantly concentrated on simulations of suspensions made of spherical particles. In this contribution, we presented an in-house developed CFD-DEM solver capable of simulations of arbitrarily-shaped particles. The conducted simulations of suspension viscosity gave a good agreement with the available experimental data even for arbitrarily-shaped particles. Moreover, we illustrated the possible usage of our solver for catalyst washcoating. The results showed a sensitivity of the coating distribution to the substrate morphology.

The source code of the solver is available at: https://github.com/MartinKotoucSourek/openHFDIB-DEM. 


\section{Acknowledgment}

The work was supported by the Grant project with No. GA19-22173S of the Czech Science Foundation, within institutional support RVO:61388998 and by the Centre of Excellence for Nonlinear Dynamic Behaviour of Advanced Materials in Engineering CZ.02.1.01/0.0/0.0/15_003/0000493 (Excellent Research Teams) in the framework of Operational Programme Research, Development and Education.

\section{References}

[1] Batchelor, G. K.: The effect of brownian motion on the bulk stress in a suspension of spherical particles. Journal of Fluid Mechanics. vol. 83 no. 1: (1977). page 97-117.

[2] Blais, B. \& Lassaigne, M. \& Goniva, C. \& Fradette, L. \& Bertrand, F.: A semi-implicit immersed boundary method and its application to viscous mixing. Comp. and Chem. Eng. vol. 85: (2016). pp. 136-146.

[3] Blazek, M. \& Zalud, M. \& Koci, P. \& York, A. \& Schlepütz, C. \& Stampanoni, M. \& Novak, V.: Washcoating of catalytic particulate filters studied by time-resolved $\mathrm{x}$-ray tomography. Chemical Engineering Journal. vol. 409: (2021). ISSN 1385-8947.

[4] Bérard, A. \& Patience, G. \& Blais, B.: Experimental methods in chemical engineering: Unresolved cfd-dem. The Canadian Journal of Chemical Engineering. vol. 98: (2020). pp. 424-440.

[5] Chauchat, J. \& Cheng, Z. \& Nagel, T. \& Bonamy, C. \& Hsu, T.-J.: SedFoam-2.0: a 3-D two-phase flow numerical model for sediment transport. Geoscientific Model Development. vol. 10 no. 12: (2017). pp. 4367-4392. doi: 10.5194/gmd-10-4367-2017.

[6] Harting, J. \& Hecht, M. \& Herrmann, H. \& McNamara, S. Computer simulation of particle suspensions. In Multifield Problems in Solid and Fluid Mechanics: pp. 113-143. Springer: Berlin: (2006).

[7] Krieger, I. \& Dougherty, T.: A mechanism for non-newtonian flow in suspensions of rigid spheres. Transactions of the Society of Rheology. vol. 3 no. 1: (1959). pp. 137-152.

[8] Mueller, S. \& Llewellin, E. \& Mader, H.: The effect of particle shape on suspension viscosity and implications for magmatic flows. Geophysical Research Letters. vol. 38: (2011).

[9] Municchi, F. \& Radl, S.: Consistent closures for Euler-Lagrange models of bi-disperse gasparticle suspensions derived from particle-resolved direct numerical simulations. Int. J. Heat and Mass Trans. vol. 111: (2017). pp. 171-190.

[10] Smuts, E. A Methodology for Coupled CFD-DEM Modelling of Particulate Suspension Rheology. PhD thesis: University of Cape Town: (2015).

[11] Sourek, M. \& Isoz, M. Recent improvements in CFD solver for fully coupled particle-laden flows. In Bodnar, T. \& Neustupa, T. \& Simurda, D., editors, Proceedings of the conference Topical Problems of Fluid Mechanics: pp. 124-131. IT CAS: Prague, Czech Republic: (2021).

[12] Studenik, O. \& Sourek, M. \& Isoz, M. Octree-generated virtual mesh for improved contact resolution in CFD-DEM coupling. In Bodnar, T. \& Neustupa, T. \& Simurda, D., editors, Proceedings of the conference Topical Problems of Fluid Mechanics: page submitted. IT CAS: Prague, Czech Republic: (2022). 